\title{
Analysis of methods for measuring the liquid level in the annular space of an oil well
}

\author{
Juriy Shinyakov, Maxim Sukhorukov, Daria Torgaeva, Andrey Soldatov", Natalia \\ Shalyapina, and Dmitriy Li \\ Tomsk State University of Control Systems and Radioelectronics, Research Institute of Space \\ Technology, 634050 Tomsk, Russia
}

\begin{abstract}
One of the most important diagnostic parameters of an oil well operation is the dynamic liquid level in the annular space. In paper the main methods for determination of the dynamic liquid level that does not require suspension of the well work and its depressurization are considered. Also the comparative analysis of these methods is given.
\end{abstract}

\section{Introduction}

Currently, oil production is carried out using sucker rod pumping units (SRPU) and electrical submersible pumping units (ESPU). Most of Russian's production well is equipped with SRP, despite the great amount of disadvantages (bulkiness of the aboveground part, incomplete wellhead sealing, limited capacity, etc.). It is also worth noting that SRP is the most suitable unit for oil production in difficult conditions (deep bedding, sand or paraffin admixture, high gas factor) which is highly topical for using in depleted wells [1].

The dynamic liquid level in the annular space of the oil well has a huge influence on the SRP operation. If the operation mode of the pump does not correspond to the rate of flow of liquid from the reservoir a decrease of dynamic level may be observed, which will lead to pressure release at the pump intake. In this case, oil degassing will lead to incomplete filling of the pump and a decrease of pump slippage. A further dynamic level decrease will lead to pump starvation. If the emergency shutdown does not actuate the operation of the pump in dry friction mode will lead to wear and damage of the parts $[2,3]$.

It is recommended to increase the depth of bore-hole pump to a dynamic level in the case of high gas con-tent in the oil-water mixture. This will lead to a pressure boost on the pump intake and a large part of the gas in the oil will be in a dissolved state. This fact will positively affect the production of the SRP. However, due to an increase in the weight of the rod string and acting floating force the mechanical and hydrodynamic resistance may increase. As a result, delivery rate and pump efficiency will be more reduced $[4,5]$.

The fluctuation of the liquid level in the annular space also has a negative effect on the pump operation because there are load variations at all nodes of the unit, rapid wear and damage of the SRP components.

There are the following methods for measuring the dynamic liquid level:

* Corresponding author: soldatov.88@bk.ru 
- $\quad$ Echometry and wavemetering;

- Thermomanometric system;

- Dynamometry and wattmetry.

\section{Echometry and wavemetering}

Echometry and wavemetering are one of the most popular methods for determining the dynamic liquid level of fluid in the annular space of an oil well. They are based on the calculation of the product of the acoustic velocity in a gaseous medium for the transit time of the signal from the emission source (wellhead) to the liquid level and back. The echometry is used for measurements in low-pressure wells, and wavemetering for wells with high pressure.

However, using this method you can get an equivocal result. Firstly, it is difficult to recognize the actual position of the liquid level on the echogram because there are noises at the receiver input. The vibrations of the tubing string, the reflection of the signal from heterogeneities in the annular space, acoustic noise of the pump also affect the acoustic signal. For this reason, the useful signal can be indistinguishable from interference. Secondly, the acoustic wave is reflected not only from the surface of the liquid, but also from any border line with a significant change in density. It is proved that at a gas-liquid mixture density of $200 \mathrm{~kg} / \mathrm{m} 3$ it is sufficient to reliably fix the response. For example, a foam column is often formed on the surface of a liquid and is a gas-liquid mixture whose density increases in depth [4]. Thirdly, the acoustic velocity in the annular well space is not constant, its value depends on the temperature, pressure, density and composition of the gas and can vary from well to well with same annular pressure even within one deposits.

Despite the disadvantages, the echometry is the main one in the oil industry because it is fairly simple to implement and inexpensive. A lot of works are devoted to the study of various techniques and tools that increase the accuracy of liquid level measurements by echometry [5-12]. For example, in algorithms for estimating the re-cording time of reflected signals are considered, structural schemes of pulse formers for sounding the annular space are proposed, which make it possible to obtain a more powerful signal that can be distinguished against background noise. Works [13, 14] describe methods for determining the speed of sound in a well: design, pipe, and benchmarking.

\section{Thermomanometric systems}

This method involves placing pressure and temperature sensors along the wellbore at a distance of $100 \mathrm{~m}$ from each other. Serial interrogation of sensors is performed to determine the dynamic liquid level. After recording the coordinates of the point "depth, pressure" in an array corresponding to the region of the well filled with gas, the correlation coefficient of the points of the entire array is calculated by least square method. The operation is repeated until the value of the coefficient does not change by more than $10 \%$ with respect to its previous value. This point indicates that the current sensor is immersed in the liquid. From now on, the "coordinates" are written to another array corresponding to the area of the well filled with liquid. After conducting a survey on the data recorded in the arrays, 2 graphs of the pressure dependence on the depth of the sensors are constructed. The point of intersection corresponds to the level of the liquid (Fig. 1). 


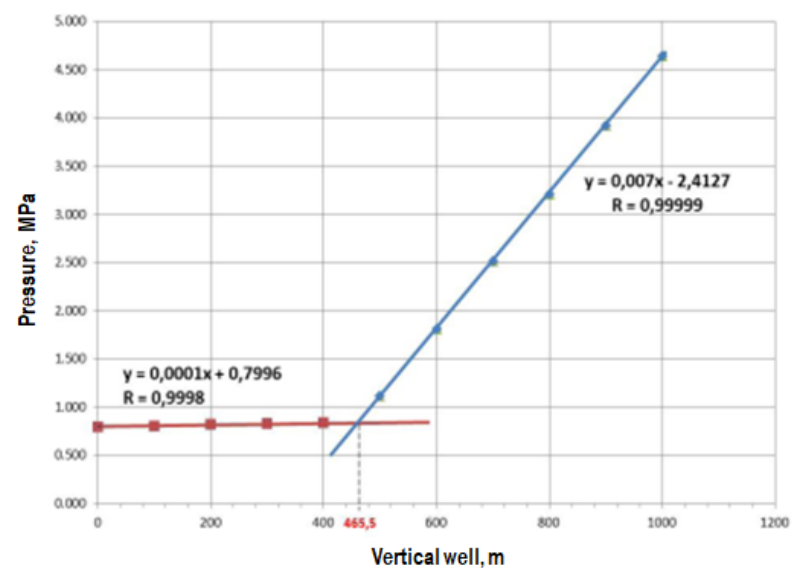

Fig.1. Determination of the liquid level in the well.

The authors of $[13,14]$ proposed to place pressure sensors along the armored vertical cable. This method is the perfect approach for ESPU (because the pump is located at the bottom of the unit, an insulated power cable leads to it). The thermomanometric system makes it possible to estimate the state of gas and liquid in the annular space in real time, however, the main disadvantage is the high cost.

A similar method for determining the dynamic level is described in [15]. Temperature sensors must be located along the entire length of the well at a distance of at least $20-40 \mathrm{~m}$ from each other. After polling all the temperature sensors, a thermogram is formed, along which the temperature gradient at each point is calculated. The point with the maximum value of the gradient will be the border line (Fig. 2).

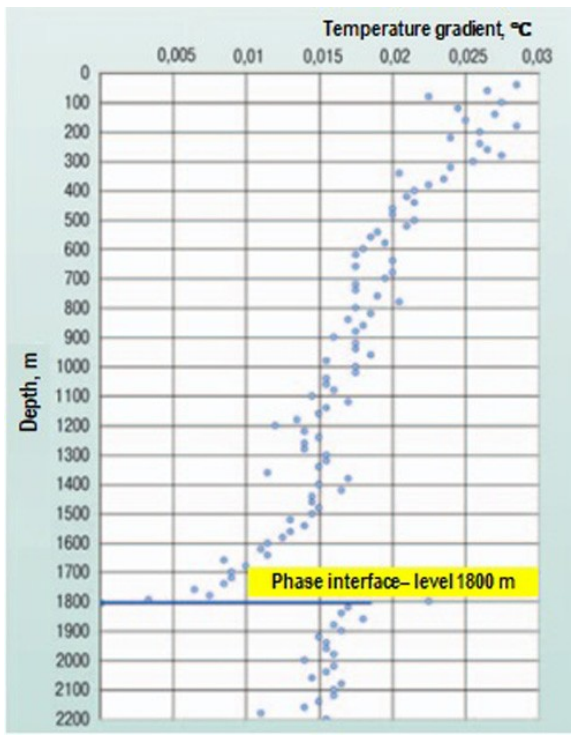

Fig. 2. Determination of the liquid level in the well according to the temperature gradient. 


\section{Dynamometry and wattmetry}

There are methods to determine the dynamic liquid level by dynamometer cards (the dependence between load on the polished rod and its stroke) and wattmeter cards (the dependence between power consumed by the motor and time).

Based on dynamometer card, it is possible to determine not only the load on the polished rod, but also the production rate of the well, and the condition of the pumping unit as a whole. The dynamometer card is the result of the interaction of a huge number of factors, so its interpretation is a very difficult task. Based on the deviation of a real dynamometer card from an ideal mathematical model, any deviations from the norm are fixed.

The article proposes a technique for calculating the change in the dynamic liquid level by an ideal dynamometer card. Initial data are the parameters of the pump itself, as well as the depth of its immersion and the value of the pressure in the pipe. This estimation method is indirect and requires large computing powers. However, the dynamic level change is usually monitored to prevent pump failure. The ingress of gas into the pump cylinder causes significant changes in the shape of the dynamometer card. In particular, when the pressure on the pump intake valve decreases, a dynamometer card of the form shown in Fig. 3a goes to the form Fig. 3b. This is caused by large gas content in the oil or a decrease in the dynamic level before the pump is received.

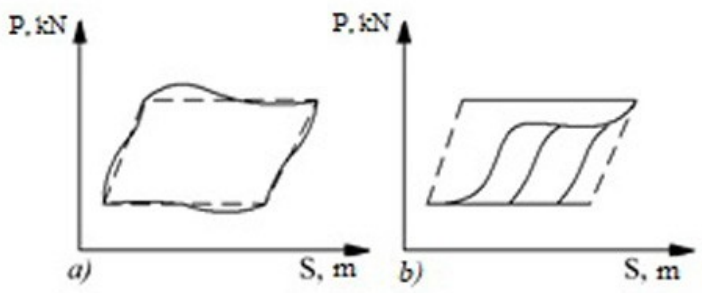

Fig. 3. Practical dynamometer cards of the SRP operation: a) normal operation with a small number of oscillations, b) gas effect.

Dynamometer cards and wattmeter cards are connected by complex non-linear dependencies. Determining the dynamic liquid level and other parameters of the pump by using wattmeter cards is an urgent task. These data allow to control the operation of the pumping unit according to the indications of a single sensor (almost all SRP are equipped with power sensors). However, a significant disadvantage of this method is the complex process of interpretation the wattmeter cards.

\section{Conclusion}

As a result, he most promising method from the considered is the method of determining the dynamic liquid level on wattmeter cards because it does not require the installation of additional equipment and allows to determine the state of the entire pumping unit by one single parameter.

The work was supported by the Ministry of Education and Science of the Russian Federation, Agreement No. 14.574.21.0157 (unique identifier RFMEFI57417X0157), within the framework of the FTP project "Research and Development in Priority Directions for the Development of the Russian Science and Technology Complex for 2014-2020". 


\section{References}

1. F. Terraneo, L. Rinaldi, M. Maggio, A. Papadopoulos, A. Leva, P.R.T. Sys. Symp. 7010370, $11(2015)$

2. S. K. Phang, J. J. Ong, R. T. C. Yeo, B. M. Chen, T. H. Lee, SIBIRCON, 722 (2010)

3. V. D. Yurkevich, SIBIRCON, 124 (2015)

4. A. I. Soldatov, A. A. Soldatov, S. I. Bortalevich, O. A. Kozhemyak, P. V. Sorokin, E. L. Loginov, Y. A. Shinyakov, M. P. Sukhorukov, SIBCON, (2017)

5. Y. V. Shulgina, A. L. Starostin, M. A. Kostina, T. S. Mylnikova, A. I. Soldatov, MEACS, (2015)

6. A. I. Soldatov, O. A. Kozhemyak, A. A. Soldatov, Yu. V.Shulgina, IOP Conf. S: Mat. S\&E, (2015)

7. Y. V. Shulgina, A. A. Soldatov, E. M. Shulgin, A. V. Kudryashova, SIBCON, (2015)

8. Yu. V. Shulgina, A. I. Soldatov, Ya. V. Rozanova, A. A. Soldatov, E. M. Shulgin, IOP Conf. S: Mat. S\&E, (2015)

9. Y. V. Shulgina, A. I. Soldatov, E. M. Shulgin, Y. V. Rozanova, M. Kroning, MEACS, (2014)

10. A. I. Soldatov, A. I. Seleznev, A. A. Soldatov, P. V. Sorokin, V. S. Makarov, Rus. J. NDT 48(5), 268 (2012)

11. A. I. Soldatov, A. I. Seleznev, A. A. Soldatov, I. I. Fiks, X. M. Kroening, Rus. J. NDT, 48(4) 255 (2012)

12. A. I. Soldatov, J. V. Chiglintseva, SIBCON, (2009)

13. H. Bodur, A. F. Bakan, M. A. Baysal, E. E. 85 (1), 45 (2003)

14. M., Borage, S. Tiwari, S. Kotaiah, IEEE T. on I. Electr. 52 (6), 1547 (2005)

15. Y. A. Shinyakov, V. D. Semenov, V. A. Kabirov, D. S. Torgaeva, M. P. SuKhorukov and R. S. Cevastyanov, SIBIRCON, 346 (2017) 